\title{
Evidence for increased intraabdominal pressure as a cause of recurrent migration of the distal catheter of a ventriculoperitoneal shunt: illustrative case
}

\author{
${ }^{*}$ Christopher Lee, BA, ${ }^{1}$ Lucinda Chiu, BS, ${ }^{1}$ Pawan Mathew, MD, ${ }^{1}$ Gabrielle Luiselli, BS, ${ }^{1}$ Charles Ogagan, BS, ${ }^{2}$ Rrita Daci, MD, ${ }^{1}$ \\ Brittany Owusu-Adjei, MD, ${ }^{1}$ Rona S. Carroll, PhD, ${ }^{1}$ and Mark D. Johnson, MD, PhD ${ }^{1,2}$ \\ 1'Department of Neurological Surgery, University of Massachusetts Medical School, Worcester, Massachusetts; and 2UMass Memorial Health Care, Worcester, Massachusetts
}

\begin{abstract}
BACKGROUND Placement of a ventriculoperitoneal (VP) shunt is an effective treatment for several disorders of cerebrospinal fluid flow. A rare complication involves postoperative migration of the distal catheter out of the intraperitoneal compartment and into the subcutaneous space. Several theories attempt to explain this phenomenon, but the mechanism remains unclear.

OBSERVATIONS The authors report the case of a 37-year-old nonobese woman who underwent placement of a VP shunt for idiopathic intracranial hypertension. Postoperatively, the distal catheter of the VP shunt migrated into the subcutaneous space on three occasions despite the use of multiple surgical techniques, including open and laparoscopic methods of abdominal catheter placement. Notably, the patient repeatedly displayed radiographic evidence of chronic bowel distention consistent with increased intraperitoneal pressure.
\end{abstract}

LESSONS In this case, the mechanism of catheter migration into the subcutaneous space did not appear to be caused by pulling of the catheter from above but rather by expulsion of the catheter from the peritoneum. Space in the subcutaneous tissues caused by open surgical placement of the catheter was permissive for this process. Patients with chronic increased intraabdominal pressure, such as that caused by bowel distention, obesity, or Valsalva maneuvers, may be at increased risk for distal catheter migration.

https://thejns.org/doi/abs/10.3171/CASE2032

KEYWORDS ventriculoperitoneal shunt; idiopathic intracranial hypertension; distal catheter migration

The ability of a ventriculoperitoneal (VP) or lumboperitoneal (LP) shunt to effectively drain the cerebrospinal fluid (CSF) relies on the proper anatomical positioning of the distal catheter within the abdominal peritoneal cavity. There are numerous reports in the literature of distal catheter migration in which the distal portion of the catheter coils along its subcutaneous path, retracting from the intraperitoneal space and preventing proper CSF drainage. ${ }^{1-12}$ In such cases, CSF accumulates in the cerebral ventricles and in the subcutaneous tissues..$^{13}$ Many of the published reports involve infants or obese patients, and a variety of theories about the mechanism underlying this phenomenon have been proposed, including a ratcheting "windlass" effect associated with movement of the head, pulling of the catheter by a shifting abdominal fat pad in obese patients during transitions from lying to standing, or increased intraabdominal pressure. $6,9,10,14$ Currently, the mechanism of distal shunt catheter migration into the subcutaneous tissues remains poorly understood.
Idiopathic intracranial hypertension $(\mathrm{IIH})$, also known as "pseudotumor cerebri," affects 0.1 to $0.2 \%$ of Americans. IIH is characterized by elevated intracranial pressure (ICP) of unknown etiology, although it is widely thought to be caused by impaired cerebral venous outflow. Risk factors for developing IIH include female sex, obesity, cerebral venous sinus stenosis, and sleep apnea. Common symptoms include chronic severe headaches and pulsatile tinnitus, although papilledema with associated vision loss is perhaps the most serious adverse complication of this disorder. Medication-refractory $\mathrm{IIH}$ requires surgical intervention to lower the ICP, relieve headaches, and/or preserve vision. Surgical options for treatment include optic nerve sheath fenestration, venous sinus stenting, or shunt placement for CSF drainage. Currently, placement of a VP or LP shunt is the most commonly used surgical treatment for $\mathrm{IIH}$. A meta-analysis of 435 patients showed that both VP and LP shunts improved symptoms in

ABBREVIATIONS CSF = cerebrospinal fluid; CT = computed tomography; ICP = intracranial pressure; IIH = idiopathic intracranial hypertension; LP = lumboperitoneal; VP = ventriculoperitoneal.

INCLUDE WHEN CITING Published January 18, 2021; DOI: 10.3171/CASE2032.

SUBMITTED September 24, 2020. ACCEPTED September 29, 2020

* C.L. and L.C. contributed equally to this work.

(C) 2021 The authors, CC BY-NC-ND 4.0 (http://creativecommons.org/licenses/by-nc-nd/4.0/), 
patients with IIH: $80 \%$ (231/287) experienced relief of headaches, $70 \%$ (107/153) experienced improvement in papilledema, and 54\% (104/ 193) reported an improvement in visual acuity. Other studies reported an improvement in headaches in $95 \%-100 \%$ of $\mathrm{IHH}$ patients after VP shunt placement. ${ }^{15-17}$

Here we present the case of a woman with IIH whose VP shunt required three surgical revisions to correct recurrent episodes of distal catheter migration into the subcutaneous space. Several surgeons employed multiple abdominal incisions and surgical techniques before this problem was resolved. The case provides strong support for the notion that pushing of the distal catheter out of the intraabdominal compartment rather than pulling from above was responsible for the recurrent episodes of distal catheter migration.

\section{Illustrative Case}

A 37 -year-old woman with a modestly elevated body mass index of 28.96 and a past medical history of hypertension, depression, anxiety, interstitial cystitis, and elective salpingectomy presented with newonset exertional headaches over the past year. She characterized the headaches as a pressure sensation involving the entire head (rated as 7-8 on a 10-point pain scale). When severe, the headaches were accompanied by blurred vision, dizziness associated with turning or standing, neck discomfort, and sensitivity to light and noise. There was no recent head or neck trauma, speech or swallowing changes, or motor abnormalities. A formal ophthalmological examination showed papilledema. A noncontrast brain magnetic resonance imaging scan showed slight prominence of the CSF surrounding the optic nerve sheaths bilaterally (Fig. $1 \mathrm{~A}$ ) in the absence of hydrocephalus or a space-occupying lesion (Fig. 1B). A partially empty sella was also noted (Fig. 1A), which, when taken together with the CSF prominence surrounding the optic nerve sheath, was suggestive of elevated ICP. A computed tomography (CT) venogram of the head showed no stenosis of the major dural sinuses. Several lumbar punctures repeatedly revealed opening pressures ranging from 25 to $30 \mathrm{~cm} \mathrm{H}_{2} \mathrm{O}$. Thus, a diagnosis of $\mathrm{IH}$ was made.

As treatment for $\mathrm{IHH}$, a shunt catheter was placed into the right lateral ventricle (Fig. 2A) from an occipital approach under stereotactic guidance. This was attached to a Codman Hakim programmable shunt valve with an integrated Rickham reservoir and distal catheter. The distal catheter was tunneled subcutaneously to a subcostal incision in
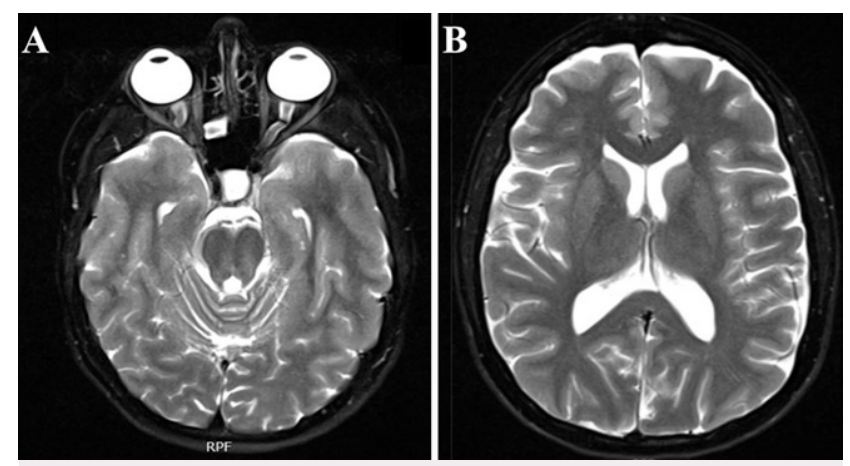

FIG. 1. Preoperative axial T2-weighted magnetic resonance images of the head. A: Slight prominence of the CSF within the bilateral optic nerve sheath complexes and a partially empty sella are observed. B: No abnormal enlargement of the lateral ventricles is observed. $\mathrm{RPF}=$ an imaging anatomical marker denoting right, posterior, foot.
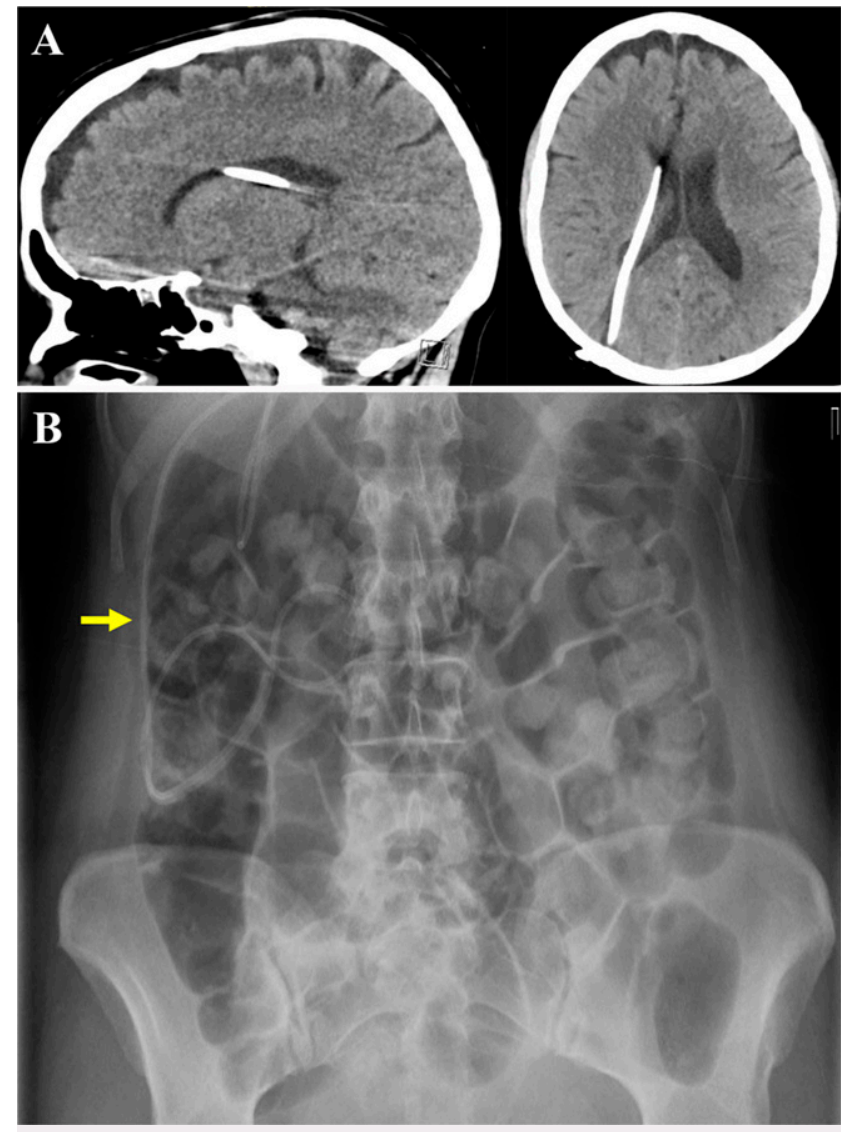

FIG. 2. Radiographic imaging after initial VP shunt system placement. A: Postoperative sagittal (left) and axial (right) noncontrast CT scans of the head. A right lateral ventricle shunt catheter is visible. B: Postoperative anterior-posterior radiograph of the abdomen and pelvis. A distal shunt catheter in the intraperitoneal cavity is seen, as indicated by the arrow. Diffuse bowel distention is observed.

the right upper quadrant of the abdomen and placed into the peritoneal cavity using a laparotomy approach (Fig. 2B). Care was taken to place a purse string suture around the peritoneal opening. The subcutaneous fat was reapproximated during closure of the wound to minimize dead space. The patient's headaches were greatly improved during the immediate postoperative period and for a period of nearly 8 weeks thereafter.

On postoperative day 54 , the patient presented to the clinic with an enlarged lump at the site of the abdominal incision. Abdominal CTand abdominal radiographs showed that the distal catheter was in the process of retracting from the peritoneum and coiling in the subcutaneous space, although the tip of the catheter was still within the peritoneum (Fig. $3 \mathrm{~A}$, far left and middle panels). She was initially observed, but the lump at the site of incision continued to enlarge over the next week. The patient was therefore taken to the operating room by neurosurgery and general surgery for revision of the distal catheter. The right upper quadrant subcostal incision was opened to retrieve the subcutaneous catheter. The catheter (which was still draining CSF) was tunneled subcutaneously to a new incision in the right lower quadrant and placed into the peritoneal cavity via open laparotomy by general surgery. The external oblique fascia was closed firmly around the catheter, and the subcutaneous tissues were 

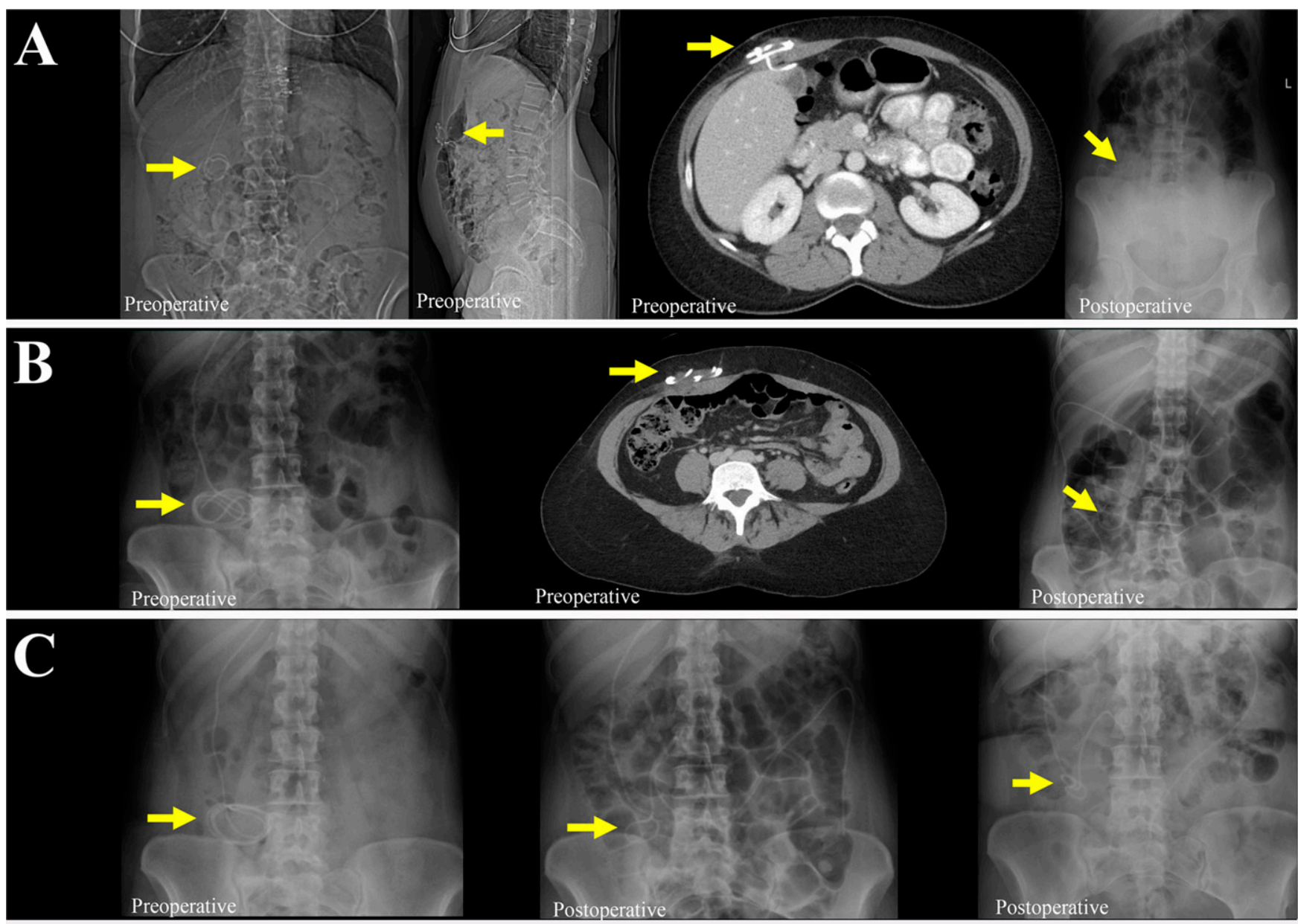

FIG. 3. A series of abdominal radiographs and CT images showing, in chronological order, three recurrent presentations of distal catheter retraction and subsequent surgical revision. Arrows indicate the location of the distal catheter. Bowel distention is observed throughout. A: First episode of retraction. Anterior-posterior (far left) and lateral (middle left) radiographs and axial noncontrast CT of the abdomen and pelvis (middle right) showing the first episode of distal peritoneal catheter retraction from the intraperitoneum. A large abdominal fat pad is not observed (middle left). Postoperative anteriorposterior radiograph of the abdomen and pelvis after surgical repositioning of the distal catheter to the right lower quadrant (far right). B: Second episode of retraction. Anterior-posterior radiograph (left) and axial noncontrast CT of the abdomen and pelvis (middle) showing the second episode of distal peritoneal catheter retraction from the intraperitoneum. Postoperative anterior-posterior radiograph of the abdomen and pelvis after surgical repositioning of the distal catheter adjacent to the previous right lower quadrant incision (right). C: Third episode of retraction. Anterior-posterior radiograph showing the third episode of distal peritoneal catheter retraction from the intraperitoneum (left), postoperative surgical repositioning of the distal catheter with creation of a gentle loop (middle), and stable positioning of the distal catheter intraperitoneally at one year after the last surgical revision (right).

reapproximated to obliterate dead space prior to closure of the skin (Fig. 3A, far right panel). Postoperatively, the patient's symptoms resolved.

On day 69 after initial shunt placement, the patient presented to the emergency department with a complaint of headaches and blurred vision. An abdominal CT scan and radiographs of the abdomen showed multiple dilated loops of bowel and retraction of the distal catheter into the subcutaneous space at the site of the new right lower quadrant incision (Fig. 3B, left and middle panels). The patient was taken to the operating room by neurosurgery and general surgery for revision of the distal shunt catheter. The right lower quadrant incision was opened to retrieve the distal catheter (which was still draining CSF), and the catheter was then placed into the peritoneal cavity laparoscopically via a separate stab incision adjacent to the right lower quadrant laparotomy incision (Fig. 3B, right panel). Postoperatively, the patient's symptoms again resolved.
On day 79 after initial shunt placement, the patient presented again to the emergency department because of headaches. Abdominal imaging revealed multiple dilated loops of bowel and migration of the distal catheter out of the peritoneal cavity and into the subcutaneous space (Fig. 3C, left panel). The patient was taken back to the operating room by neurosurgery and general surgery for a third revision. To prevent another episode of catheter migration, the surgical team reopened the right lower quadrant laparotomy incision to retrieve the coiled loops of distal catheter. They then formed a gentle loop in the catheter and sutured the looped catheter to the external oblique fascia for strain relief (Fig. 3C, middle panel). Next, the catheter was tunneled through the subcutaneous tissue to a stab incision near the midline (at a significant distance from the laparotomy incision) and placed laparoscopically into the peritoneal cavity. The subcutaneous tissues at the 
laparotomy incision were reapproximated to eliminate dead space prior to closure of the skin. Postoperatively, the patient's symptoms resolved.

On day 155 after initial shunt placement, the patient presented to the clinic for evaluation. An abdominal radiograph showed that the distal catheter had partially retracted and formed additional coils in the subcutaneous space at the site where the catheter loop had been secured to the fascia (not shown). However, subsequent imaging over the next few months revealed no further distal catheter migration. One year from the last shunt revision, the distal shunt catheter was noted to remain within the peritoneal cavity (Fig. $3 \mathrm{C}$, right panel), and the patient had no signs or symptoms of elevated ICP.

\section{Discussion}

\section{Observations}

In general, shunt complications impose a high cost on patients and the healthcare system. One study showed that the need for shunt revision surgeries yielded a fivefold increase in the financial cost of hydrocephalus treatment. ${ }^{18} \mathrm{~A}$ recent review by Satti et al. proposed a reevaluation of CSF drainage as the standard treatment for IIH because of the high complication rates. ${ }^{19}$ Meanwhile, costs for patients include extended hospital stays, employment disruption, stress on caregivers, and the risk of intraoperative and postoperative complications. Thus, it is important to understand the underlying mechanisms of distal catheter migration so that this complication can be avoided.

In a recent meta-analysis of 358 studies, the rates of surgical revision of CSF shunts were $7.6 \%$ and $33 \%$, respectively, for major (shunt infection, tonsillar herniation, subdural hematoma, and CSF fistula) and minor complications (abdominal pain, valve dysfunction, radicular pain, shunt disconnection, shunt malposition, low-pressure headaches, CSF leak, and catheter migration). ${ }^{19}$ These numbers combine VP and LP shunts, although distal catheter migration occurs at similar rates between VP and LP shunt systems. ${ }^{15}$ Distal catheter migration occurred in $5 \%$ of properly placed CSF shunts ${ }^{15}$ and was found to represent $7.4 \%$ of all complications. ${ }^{20}$

Here we describe the case of a 37-year-old woman with a history of IIH who required three shunt revision surgeries within 79 days of her initial VP shunt placement because the distal catheter repeatedly migrated out of the peritoneum and into the subcutaneous space. This uncommon complication occurred on days 54,69, and 79 after initial VP shunt placement, despite the use of multiple incisions during separate surgeries that were performed by different surgeons over a period of several months. Several theories have been proposed to account for the phenomenon of distal catheter migration into the subcutaneous space. They can be grouped into two major categories: pulling (e.g., the windlass effect, abdominal fat pad shift, repeated head turning, etc.) and pushing (e.g., increased intraabdominal pressure, as may be found in obese patients). ${ }^{5-10,14,21}$

The circumstances surrounding this case strongly suggest that increased intraabdominal pressure was a major contributing factor in recurrent distal catheter migration. The windlass effect ${ }^{6,9,10,14}$ is unlikely to have taken place, given that partial distal catheter migration occurred despite placing a tethered loop for strain relief and using a laparoscopic trocar-based approach to place the catheter. Furthermore, the fat pad shift ${ }^{6}$ explanation is unlikely because the patient was only mildly overweight (body mass index 28.96, Fig. 3A, middle left panel). Instead, it is more likely that the catheter was being pushed out of the peritoneum. In support of this notion was the striking finding of multiple dilated loops of bowel on every radiographic study of the abdomen obtained during her repeated shunt revisions. Of note, a retrograde pyelogram taken prior to initial shunt placement, but while the patient was symptomatic from her $\mathrm{IIH}$, revealed evidence of bowel distention (not shown). This chronic crowding of the intraabdominal contents is consistent with the presence of increased intraabdominal pressure. This may have contributed to a decrease in intracranial venous outflow ${ }^{22,23}$ that would explain why a nonobese woman with no evidence of intracranial venous stenosis presented with signs and symptoms of $\mathrm{IH}$.

Although the mechanism underlying distal catheter migration from within the peritoneum may involve high intraperitoneal pressure in this case, such a mechanism has not been rigorously examined in the literature previously. However, there have been numerous studies reporting that elevated intraperitoneal pressure can lead to distal catheter perforation of the bowel. Bowel perforation constitutes $0.1-1.0 \%$ of VP and LP shunt complications. ${ }^{24}$ It presents clinically due to extrusion of the distal catheter most often through the anus $(68.6 \%)$, scrotum $(22 \%)$, umbilicus $(6.9 \%)$, and vagina $(3.2 \%){ }^{25}$ Numerous case reports have also described extrusion from these and other locations: 21 peroral, ${ }^{12} 57$ transanal, ${ }^{26} 3$ vaginal, ${ }^{27} 2$ transabdominal, ${ }^{28} 1$ transurethral, ${ }^{29}$ and 2 transscrotal. ${ }^{30}$ Given that all patients presented in a delayed fashion, Al Fauzi et al. hypothesized that it must be due to a chronic disease process rather than acute injury at the time of the procedure. ${ }^{12}$ Additionally, de Aquino et al. postulated that bowel peristaltic movements and increased intraabdominal pressures supply a constant and high enough source of mechanical pressure to induce perforation. ${ }^{25}$ Fortunately, there was no evidence of bowel perforation or infection in this case.

\section{Lessons}

VP shunt failure due to upward migration of the distal catheter is a rare complication, and the underlying mechanisms are poorly understood. In this case, the major contributing factor leading to recurrent distal catheter migration was increased intraabdominal pressure due to chronic bowel distension rather than obesity. Space in the subcutaneous tissues caused by open surgical placement of the catheter was permissive for this process. It is important to consider whether patients with chronic increased intraabdominal pressure, such as extensive bowel distention, obesity, or excessive Valsalva maneuvers, may be at increased risk for distal shunt catheter migration.

Ultimately, this problem was resolved by fastening a loop of catheter to the fascial wall, similar to the technique that Browd et al. used to secure the proximal shunt catheter. ${ }^{31}$ The concurrent use of laparoscopy to minimize the presence of a potential space in the subcutaneous tissues was also important. In retrospect, rerouting the distal catheter early in this process so that it did not traverse the dead space present at the sites of the open laparotomy incisions may have reduced some of the episodes of recurrent catheter migration.

Our case report is limited in scope because it describes observations made from an individual female patient with IIH. However, this case, in combination with other reports of distal catheter migration in the literature, strongly implicates a role for increased intraabdominal pressure in this phenomenon.

\section{Acknowledgments}

This research was funded by grant support (Grant no. 1R01NS106985-01) from the National Institutes of Health to M.D.J.

\section{References}

1. Cho KR, Yeon JY, Shin HJ. Upward migration of a peritoneal catheter following ventriculoperitoneal shunt. J Korean Neurosurg Soc. 2013;53(6):383-385.

2. Martínez-Lage JF, Poza M, Izura V. Retrograde migration of the abdominal catheter as a complication of ventriculoperitoneal shunts: the fishhook sign. Childs Nerv Syst. 1993;9(7):425-427. 
3. Garling RJ, Sood S, Harris CA. Avoiding the pocket: a case report of coiling of distal shunt catheter into subcutaneous pocket. Int $J$ Surg Case Rep. 2017;41:61-64.

4. Gatto LA, Mathias R, Tuma R, et al. Rare complication of ventriculoperitoneal shunt: catheter protrusion to subcutaneous tissue - case report. Surg Neurol Int. 2016;7(suppl 44): S1142-S1146.

5. Morrison JF, Sung KE, Bergman AM, et al. A novel solution to reduce the complications of distal shunt catheter displacement associated with obesity. J Neurosurg. 2010;113(6):1314-1316.

6. Nagasaka T, Inao S, Ikeda H, et al. Subcutaneous migration of distal ventriculoperitoneal shunt catheter caused by abdominal fat pad shift--three case reports. Neurol Med Chir (Tokyo). 2010; 50(1):80-82.

7. Thaker NG, Mammis A, Yanni DS, et al. Distal subgaleal-peritoneal shunt migration into the abdominal wall with subsequent formation of a pre-peritoneal pseudocyst: a rare complication. J Surg Case Rep. 2010;2010(7):9.

8. Nakahara K, Shimizu S, Oka H, et al. Migration of the distal end of a ventriculoperitoneal shunt into the abdominal wall in an obese patient: case report. Neurol Med Chir (Tokyo). 2009;49(10): 490-492.

9. Kim KJ, Wang KC, Cho BK. Proximal migration and subcutaneous coiling of a peritoneal catheter: report of two cases. Childs Nerv Syst. 1995;11(7):428-431.

10. Heim RC, Kaufman BA, Park TS. Complete migration of peritoneal shunt tubing to the scalp. Childs Nerv Syst. 1994;10(6): 399-400.

11. Ferraresi S, Griffini C, Torcello L, et al. Duplicated peritoneal catheter as a cause of shunt malfunction. Case report. Neurosurg Rev. 1991;14(2):149-150.

12. Al Fauzi A, Suryaningtyas W, Wahyuhadi J, et al. Upward migration and peroral extrusion of a peritoneal shunt catheter: case report and review of the literature. Surg Neurol Int. 2017; 8:178.

13. Abode-lyamah KO, Khanna R, Rasmussen ZD, et al. Risk factors associated with distal catheter migration following ventriculoperitoneal shunt placement. J Clin Neurosci. 2016;25:46-49.

14. Scott M, Wycis HT, Murtagh F, et al. Observations on ventricular and lumbar subarachnoid peritoneal shunts in hydrocephalus in infants. J Neurosurg. 1955;12(2):165-175.

15. McGirt MJ, Woodworth G, Thomas G, et al. Cerebrospinal fluid shunt placement for pseudotumor cerebri-associated intractable headache: predictors of treatment response and an analysis of long-term outcomes. J Neurosurg. 2004;101(4):627-632.

16. Burgett RA, Purvin VA, Kawasaki A. Lumboperitoneal shunting for pseudotumor cerebri. Neurology. 1997;49(3):734-739.

17. Johnston I, Paterson A. Benign intracranial hypertension. II. CSF pressure and circulation. Brain. 1974;97(2):301-312.

18. Ahmed RM, Wilkinson M, Parker GD, et al. Transverse sinus stenting for idiopathic intracranial hypertension: a review of 52 patients and of model predictions. AJNR Am J Neuroradiol. 2011;32(8):1408-1414.

19. Satti SR, Leishangthem L, Chaudry MI. Meta-analysis of CSF diversion procedures and dural venous sinus stenting in the setting of medically refractory idiopathic intracranial hypertension. AJNR Am J Neuroradiol. 2015;36(10):1899-1904.
20. Sinclair AJ, Kuruvath $S$, Sen D, et al. Is cerebrospinal fluid shunting in idiopathic intracranial hypertension worthwhile? A 10year review. Cephalalgia. 2011;31(16):1627-1633.

21. Eljamel MS, Sharif S, Pidgeon $C N$. Total intraventricular migration of unisystem ventriculo-peritoneal shunt. Acta Neurochir (Wien). 1995;136(3-4):217-218.

22. Friedman DI, Liu GT, Digre KB. Revised diagnostic criteria for the pseudotumor cerebri syndrome in adults and children. Neurology. 2013;81(13):1159-1165.

23. Sugerman HJ, Felton WL 3rd, Salvant JB Jr, et al. Effects of surgically induced weight loss on idiopathic intracranial hypertension in morbid obesity. Neurology. 1995;45(9):1655-1659.

24. Vinchon M, Baroncini M, Laurent T, Patrick D. Bowel perforation caused by peritoneal shunt catheters: diagnosis and treatment. Neurosurgery. 2006;58(1 suppl):ONS76-ONS82.

25. de Aquino HB, Carelli EF, Borges Neto AG, et al. Nonfunctional abdominal complications of the distal catheter on the treatment of hydrocephalus: an inflammatory hypothesis? Experience with six cases. Childs Nerv Syst. 2006;22(10):1225-1230.

26. Sarkari A, Borkar SA, Mahapatra AK. Anal extrusion of migrated ventriculo-peritoneal shunt catheter: an unusual complication and review of literature. Asian J Neurosurg. 2016;11(4):459.

27. Bonfield CM, Weiner GM, Bradley MS, et al. Vaginal extrusion of a ventriculo-peritoneal shunt catheter in an adult. J Neurosci Rural Pract. 2015;6(1):97-99.

28. Panigrahi S, Mishra SS, Das S, et al. Spontaneous extrusion of peritoneal catheter of ventriculoperitoneal shunt through the intact abdominal wall: report of two cases. J Pediatr Neurosci. 2012; 7(3):228-230.

29. de Aguiar GB, Mizrahi C, Aquino JH, et al. Urethral extrusion of a peritoneal catheter in a patient with a neobladder: a rare complication of shunt insertion. Neuropediatrics. 2011;42(3):124-127.

30. Ozveren MF, Kazez A, Cetin H, et al. Migration of the abdominal catheter of a ventriculoperitoneal shunt into the scrotum--case report. Neurol Med Chir (Tokyo). 1999;39(4):313-315.

31. Browd SR, Ragel BT, Gottfried ON, et al. Failure of cerebrospinal fluid shunts: part I: obstruction and mechanical failure. Pediatr Neurol. 2006;34(2):83-92.

\section{Disclosures}

The authors report no conflict of interest concerning the materials or methods used in this study or the findings specified in this paper.

\section{Author Contributions}

Conception and design: Johnson, Lee, Ogagan. Acquisition of data: Johnson, Mathew. Analysis and interpretation of data: Johnson, Chiu, Mathew, Daci. Drafting the article: Johnson, Lee, Chiu, Mathew, Ogagan, Daci. Critically revising the article: Johnson, Lee, Chiu, Mathew, Luiselli, Daci, Owusu-Adjei. Reviewed submitted version of manuscript: all authors. Approved the final version of the manuscript on behalf of all authors: Johnson. Administrative/technical/material support: Johnson, Lee, Mathew, Carroll. Study supervision: Johnson.

\section{Correspondence}

Mark D. Johnson: University of Massachusetts Medical School, Worcester, MA. mark.johnson3@umassmemorial.org. 